\title{
Accuracy of self-perceived risk perception of breast cancer development in Iranian women
}

\author{
Karimollah Hajian-Tilaki ${ }^{1,2}$ and Maryam Nikpour ${ }^{3^{*}}$
}

\begin{abstract}
Background: The accuracy of subjective risk perception is a matter of concern in breast cancer development. The objective of this study was to evaluate the accuracy of self-perceived risk assessment of breast cancer development and compared to actual risk in Iranian women.

Methods: The demographic, clinical, and reproductive characteristics of 800 women aged 35-85 years were collected with an in-person interview. The self-perceived risk and the actual risk were assessed using the visual analog scale (VAS) and he Gail model respectively. Gail's cutoff of 1.66\% risk was used to categorize the estimated 5-year actual risk as low/average risk $(<1.66 \%)$ and high risk $(\geq 1.66)$. In low/average risk, if the self-perceived risk $>$ actual risk, then individuals were considered as overestimating. Similarly, in high-risk women, if the perceived risk < actual risk, then, the subjects were labeled as under-estimate; otherwise, it was labeled as accurate. The Kappa statistics were used to determine the agreement between self-perceived risk and actual risk. ROC analysis was applied to determine the accuracy of self-perceived risk in the prediction of actual risk.

Results: The perceived risk was significantly higher than actual risk $(p=0.001,0.01$ for 5 -year and lifetime risk respectively). Both in low and high-risk groups about half of the women over-estimate and underestimate the risk by subjective risk perception. For a 5 -year risk assessment, there was no agreement between perceived risk and actual risk (Kappa $=0.00, p=0.98)$ but a very low agreement between them in lifetime risk assessment (Kappa $=0.09, p=0.005)$. The performance of accuracy of risk perception versus actual risk was very low (AUC $=0.53,95 \% \mathrm{Cl} 0.44-0.61$ and $\mathrm{AUC}=0.58,95 \% \mathrm{Cl} 0.54-0.62$ for the 5-year risk and lifetime risk respectively).
\end{abstract}

Conclusion: The clinical performance of risk perception based on VAS is very poor. Thus, the efforts of the public health education program should focus on the correct perception of breast cancer risk among Iranian women.

Keywords: Self-perceived risk, Actual risk, Five-year risk, Lifetime risk, Gail's model

\section{Background}

Breast cancer $(\mathrm{BC})$ is the most common cancer [1] and the second cause of death (after lung cancer) in women worldwide [2]. BC is one of the most expensive cancers in the world with a financial burden of about $\$ 88$ billion annually [3]. In Iran, BC is the most common cancer and it is composed of a quarter of malignancies. The age of

*Correspondence: Maryamnikpour19@yahoo.com

${ }^{3}$ Non-Communicable Disease Research Center, Health Research Institute,

Babol University of Medical Sciences, Babol, Iran

Full list of author information is available at the end of the article involvement by breast cancer in Iranian women is about 10 years less than other developed countries [3].

Incidence and mortality rates of $\mathrm{BC}$ are increasing in Iranian women $[4,5]$. The increasing incidence in developing counties mainly attributed to changes in lifestyles and reproductive behaviors [6-10]. The majority of women with $\mathrm{BC}$ are diagnosed at a higher stage of the disease that is corresponded with a high mortality rate [11-13]. To establish a preventive strategy for early detection, the risk assessment is primarily interested in $\mathrm{BC}$ from a public health perspective that can be used as a preventive strategy for early detection. Several methods 
of risk assessment of $\mathrm{BC}$ have been suggested [14-17]. They can be categorized as objective risk assessment or actual risk and subjective risk perception. The Gail model was mainly adopted for quantitative assessment of BC development. It predicts the actual risk as an objective measure based on the profile of risk factors [15]. The second method is the self-perceived risk perception that is based on the subjective matter on the visual analog scale (VAS). Another method of perceived risk assessment is the health belief model (HBM) scale to measure the perceived risk in different domains (susceptibility, seriousness, benefit, barriers, self-efficacy/confidence, and health motivation) [17]. The performance of HBM on screening behaviors has been acknowledged in several investigations [13, 17-19].

The self-perceived risk perception based on the analog scale is a simple method that may use in clinical practices for purpose of screening. It can be motivates the women to undergo the screening program such as breast selfexamination (BSE), breast clinical examination (BCE) and mammography. However, subjective risk perception is likely to be either optimistic or pessimistic [20-23]. Thus, inaccurate risk perception may induce psychological symptoms, in particular in the case of over-estimate. It may concern that women either underestimate or overestimate their risk based on this subjective matter. Meanwhile, the subjective risk assessment depends on the culture and environmental conditions. Its accuracy is a matter of concern, and data in this regard is sparse in the Asian population, particularly in the Islamic Republic of Iran. This study aimed to evaluate the accuracy of selfperceived risk assessment compared to the objective risk and to identify characteristics of individuals who do not accurately perceive their risk of developing $\mathrm{BC}$.

\section{Methods}

\section{Study design and subjects}

This cross-sectional study was conducted in Babol, northern Iran in 2018. Data were reanalyzed for risk assessment of breast cancer development. A total of 800 women at age of 35-85 years participated in the study. In comparing between the mean of objective risk by Gail model and perceived risk by VAS scale in our pair samples, the allocated sample size could detect the effect size of 0.1 with $95 \%$ confidence interval and $80 \%$ power. A cluster sampling technique was used to recruit individuals in the study in the randomly selected communitybased clusters and also the outpatient clinic. The details of sampling methods were described elsewhere [12]. In brief, 20 randomly selected community-based clusters and 3 outpatient clinics in the major educational hospitals were used to select samples. Individuals with a previous history of a histologically confirmed diagnosis of breast cancer were excluded. The study protocol was approved by the institutional board of the National Institute of Medical Research Development (NIMAD), Tehran, Iran.

\section{Data and instruments}

The data of demographic, clinical and reproductive characteristics were collected with in-person interviews using a standard questionnaire of breast cancer assessment. This questionnaire was developed by US National Cancer Institute and it is included patient eligibility, demographic and para-clinical findings of biopsy and family history [24]. More specifically, these data composed of age, educational level, marital status and occupation, the first menstrual age, age at first childbirth. Also there are information such as history of breast cancer in close family members (mother, daughters, and sisters), history of biopsy of the breast, number of biopsies, identify of dysplasia in the biopsy. We also used the Persian version of the breast cancer awareness measure (BCAM) questionnaire [25]. This scale originally developed by UK cancer center and validated specific to $\mathrm{BC}$ awareness [26]. The Persian version of this scale was investigated by Heidari and Feizi [25] that showed a high test-retest reliability $(\mathrm{ICC}=0.84)$ and internal consistency (Cronbach's alpha $=0.88$ ). This scale composed of the knowledge of women in breast cancer risk factors and the sign and symptoms of breast cancer. It has 14 items of multiplechoice scale to measure the awareness of risk factors and 8-item scale to assess the signs and symptoms with binary response. The score of both measures was categorized as low ( $\leq$ the average scale) and high ( $>$ average of scale). The self-perceived 5-year and lifetime risk were assessed using a visual analog scale (VAS) ranging from 0 to 100 . The participants showed the appropriate point in this scale for their risk perception of breast development for the next 5-years and also lifetime risk. Meanwhile, the objective risk/actual risk was calculated from the modified Gail's model. This model was automated with an interactive online program based on clinical and reproductive data of individuals' risk profiles. This program computes the actual 5-year and lifetime risk and also the average risk with similar age of individuals in the population of women. The details of this risk calculation were explained elsewhere [12].

\section{Statistical analysis}

We used SPSS software version 18.0 for data analysis. The descriptive statistics for quantitative data of selfperceived and actual risk were shown as median and quartiles and for categorical data as frequencies and percentages. The Wilcoxon related sample test was used to compare the mean of perceived risk and actual risk. The 
Gail's cutoff criterion of $1.66 \%$ risk was used to categorize the estimated 5-year risk/actual risk of participants as low/average risk $(<1.66 \%)$ and high risk $(\geq 1.66)$. A similar classification was applied for lifetime risk at a cutoff point of $10 \%$ ( $<10 \%$ vs. $\geq 10 \%$ risk), According to objective risk status (low/average and high risk), among the participants with low/average risk, if the self-perceived risk > actual risk, then the perceived risk was categorized as over-estimate. While among individuals with high actual risk, if the perceived risk < actual risk, then, the subjects were as under-estimate; otherwise, it was labeled as accurate. The frequencies and percentage of over-estimate and under-estimate of self-perceived risk was calculated according to actual risk status. The association of individuals' characteristics with over-estimate and under-estimate of self-perceived risk was determined by the cross-classification of data using the Chi-square test. Based on the categorization of both perceived risk and actual risk (cutoff value of $1.66 \%$ of the 5 -year risk and $10 \%$ of lifetime risk), the Kappa statistics as an index of agreement and the $p$ value of McNemar test was calculated. Moreover, ROC analysis was applied to identify the diagnostic value of perceived risk to classify correctly the actual risk based on the cutoff points of $1.66 \%$ and $10 \%$ for actual 5-year risk and actual lifetime risk respectively. The area under the curve and its $p$ value were calculated. All tests were two-sided and a $p$ value less than 0.05 was considered as significant level.

\section{Results}

The mean age (SD) of participants was 47.63 (10.46) years and the majority of individuals were at age $35-49$ years $(61.5 \%)$ and the minority $(7.1 \%)$ was at age $65-85$ and the rest were at age of 50-64 years. The majority of women were at an educational level of high school or higher $(81.0 \%)$ and housewife $(74.1 \%)$. A few participants $(7 \%)$ had a family history of breast cancer in first-degree relatives. Almost, $84.9 \%$ were married and $6.6 \%$ single and the remainders were either divorced or widow (8.5\%). About half of participants had a low level of knowledge of breast cancer risk factors and $34.8 \%$ had a low level of awareness of signs and symptoms of breast (Table 1). Table 2 shows that the mean of self-reported perceived 5-year risk and lifetime risk was significantly higher than actual risk $(9.19 \pm 16.1$ vs. $0.89 \pm 0.89, p=0.001$, and $14.87 \pm 20.79$ vs. $8.87 \pm 3.84$ vs. $p=0.01$ respectively). While the median of perceived risk was rather lower than actual risk ( $0 \%$ vs. $0.7 \%$, and $5.0 \%$ vs. $8.3 \%$ ). But the third quartile (Q3) of perceived risk much greater than the actual risk for both 5 -year and lifetime risk. Table 3 indicates that among participants with low or average 5-year risk for disease, roughly $45.7 \%$ of women, their perceived
Table 1 Characteristics of study subjects

\begin{tabular}{|c|c|c|}
\hline Characteristics & $\mathbf{N}$ & $\%$ \\
\hline \multicolumn{3}{|l|}{ Age (year) } \\
\hline $35-49$ & 492 & 61.5 \\
\hline $50-64$ & 251 & 31.4 \\
\hline$\geq 65$ & 57 & 7.1 \\
\hline \multicolumn{3}{|l|}{ 1st menstrual age (year) } \\
\hline$<12$ & 119 & 15.1 \\
\hline$\geq 12$ & 667 & 84.9 \\
\hline \multicolumn{3}{|l|}{ Age at 1st birth (year) } \\
\hline$<20$ & 249 & 33.2 \\
\hline $20-24$ & 317 & 42.3 \\
\hline $25-29$ & 141 & 18.8 \\
\hline$\geq 30$ & 43 & 5.7 \\
\hline \multicolumn{3}{|c|}{ Breast cancer at 1st degree of relatives } \\
\hline No & 742 & 93.0 \\
\hline Yes & 58 & 7.0 \\
\hline \multicolumn{3}{|l|}{ No of biopsy } \\
\hline None/not applicable & 744 & 93.0 \\
\hline$\geq 1$ & 56 & 7.0 \\
\hline \multicolumn{3}{|l|}{ Education } \\
\hline Illiterate & 78 & 9.8 \\
\hline Primary & 154 & 19.3 \\
\hline High school & 393 & 49.1 \\
\hline University & 175 & 21.9 \\
\hline \multicolumn{3}{|l|}{ Occupation } \\
\hline Housewife & 593 & 74.0 \\
\hline Employee & 175 & 21.9 \\
\hline Retired & 32 & 4.0 \\
\hline \multicolumn{3}{|l|}{ Marital status } \\
\hline Single & 53 & 6.6 \\
\hline Married & 679 & 84.9 \\
\hline Divorced/widow & 68 & 8.5 \\
\hline \multicolumn{3}{|l|}{ Knowledge of risk factor } \\
\hline Low & 419 & 52.6 \\
\hline High & 377 & 47.4 \\
\hline \multicolumn{3}{|c|}{ Awareness of signs and symptoms of $B C$} \\
\hline Low & 275 & 34.8 \\
\hline High & 516 & 65.2 \\
\hline
\end{tabular}

risk was over-estimated. While among high risk, 54.1\% of participants under-estimated in their perceived risk. Based on the objective/actual 5-year risk assessment (Gail criteria) 61 women (7.5\%) were at high risk. In contrast, for perceived risk with a similar cutoff value was $366(45.8 \%)$. This also shows that higher level 5 -year perceived risk compared to the actual risk for BC. The Kappa statistics across data in Table 3 show that there is no agreement between perceived risk and actual risk (Kappa $=0.00, p=0.98)$. 
Table 2 The mean (SD) and the quartiles of self-perceived risk and estimated actual risk of women BC development in study sample

\begin{tabular}{lllll}
\hline & Perceived 5-year risk & Perceived lifetime risk & Actual 5-year risk & Actual lifetime risk \\
\hline Mean (SD) \% & $9.19(16.1)$ & $14.87(20.79)$ & $0.89(0.89)$ & $8.87(3.84)$ \\
Q1\% & 0.00 & 0.00 & 0.50 & 6.82 \\
Q2 (Median) \% & 0.00 & 5.00 & 0.70 & 8.30 \\
Q3\% & 10.00 & 20.00 & 1.10 & 10.30 \\
\hline
\end{tabular}

Table 3 The self-perceived 5-year risk of BC development according to corresponded estimated actual 5-year risk

\begin{tabular}{llll}
\hline $\begin{array}{l}\text { Perceived 5-year } \\
\text { risk }\end{array}$ & Actual 5-year risk & All \\
\cline { 2 - 3 } & $<\mathbf{1 . 6 6 \%}$ risk & $\mathbf{2 1 . 6 6 \% \text { risk }}$ & \\
\hline$<1.66 \%$ & $401(54.3)$ & $33(54.1)$ & $434(54.3)$ \\
$\geq 1.66 \%$ & $338(45.7)$ & $28(45.9)$ & $366(45.8)$ \\
All & $739(100)$ & $61(100)$ & $800(100)$ \\
\hline
\end{tabular}

Kappa $=0.00 ; p=0.98$

The data show the frequencies and percentage in the parenthesis

Table 4 The self-perceived lifetime risk of BC development according to corresponded estimated actual lifetime risk

\begin{tabular}{llll}
\hline $\begin{array}{lll}\text { Perceived lifetime } \\
\text { risk }\end{array}$ & \multicolumn{2}{l}{ Actual lifetime risk } & All \\
\cline { 2 - 3 } & $<\mathbf{1 0 \%}$ risk & $\mathbf{2 1 0 \% \text { risk }}$ \\
\hline$<10 \%$ & $316(55.0)$ & $99(44.0)$ & $415(51.9)$ \\
$\geq 10 \%$ & $259(45.0)$ & $126(56.0)$ & $385(48.1)$ \\
All & $576(100)$ & $225(100)$ & $800(100)$ \\
\hline
\end{tabular}

Kappa $=0.09 ; p=0.005$

The data show the frequencies and percentage in the parenthesis

Table 4 shows that women at high lifetime risk, $44.0 \%$ times under-estimated their risk while low or averagerisk subjects, $45.0 \%$ of times over-estimated their risk in BC development. Based on actual lifetime risk, 225 (28.1\%) subjects were labeled as high risk but this figure for perceived risk was $385(48.1 \%)$ individuals. The Kappa statistics were $0.09(p=0.005)$ which shows a low level of agreement between perceived risk and actual risk (Kappa $=0.09, p=0.005)$.

Table 5 also presents that among women with low/ average risk, the percentage of over-estimate of perceived risk was significantly higher among younger $(p=0.05)$, the high level of knowledge of risk factors $(p=0.002)$ and symptoms and signs $(p=0.004)$ were associated with the higher education level $(p=0.04)$. While in a high-risk group, the data did not show a clear pattern of under-estimate of perceived risk with individuals' characteristics.
Table 6 and Fig. 1 in panel (a) and (b) display the corresponded accuracy and the location of ROC curves of perceiving risk in the prediction of actual 5-year and lifetime risk. The AUCs were 0.53 (95\% CI 0.44-0.61; $p=0.47)$ and 0.58 (95\% CI $0.54-0.62, p=0.001)$ for perceived risk in prediction of actual 5-year risk and lifetime risk respectively. The perceived risk has appeared with very low sensitivity and specificity $(\operatorname{Sen}=45.9 \%$ and $\mathrm{Sp}=54.3 \%$ for 5 -year risk and $\mathrm{Sen}=56 \%, \mathrm{Sp}=55 \%$ for lifetime risk). These findings also show the low performance for accuracy of self-perceived risk on VAS in the prediction of the actual risk. Additionally, the perceived risk in VAS yielded low performance in positive predicted value (PPV) but relatively high in negative predicted value (NPV).

\section{Discussion}

The study was conducted to assess the perceived risk of breast cancer compared to the actual risk in 800 Iranian women. Finding of the study showed that the perceived risk of breast cancer in women with low and high risk of breast cancer was significantly higher than the actual risk. In other words, study women overestimated of breast cancer risk than routine. This finding indicates a significant pessimism in breast cancer perception.

The results of the study were in line with the reports of Ceber et al. [21], John and et al. [23], and Aduayi and et al. [27]. Jones and colleagues found that the estimated risk among 3000 Australian women was higher than the real risk [23]. However the results of the study were inconsistent with the findings of studies by Chung et al. [20] and Katapodi et al. [29]. A study by Chung et al. [20] found that most Korean women (51\%) were optimistic about their relative risk of breast cancer and only $2.3 \%$ had pessimistic perception. In the study of Katapodi and et al. [29] an optimistic and pessimistic perception of the risk of breast cancer was $40 \%$ and $10 \%$, respectively. Optimistic means that fewer negative events and more positive events happen to themselves than others [20]. There are probably several reasons for this inconsistency. In Chung et al. study, samples were selected from health centers. Whether, in our study samples were selected from the community 
Table 5 The distribution of over-estimate and under-estimate with respect to individuals' characteristics of women among low/average risk and high risk groups

\begin{tabular}{|c|c|c|c|c|c|c|}
\hline \multirow[t]{2}{*}{ Characteristics } & \multicolumn{3}{|c|}{ Low/average risk $(n=739)$} & \multicolumn{3}{|l|}{ High risk $(n=61)$} \\
\hline & Accurate n (\%) & Over-estimate $n(\%)$ & $p$ value & Accurate n (\%) & $\begin{array}{l}\text { Under- } \\
\text { estimate n (\%) }\end{array}$ & $p$ value \\
\hline Age group (year) & & & 0.05 & & & 0.63 \\
\hline $35-49$ & $241(49.9)$ & $242(50.1)$ & & $3(33.3)$ & $6(66.7)$ & \\
\hline $50-64$ & $125(56.8)$ & $95(43.2)$ & & $14(45.2)$ & $17(54.8)$ & \\
\hline Relatives $\geq 65$ & $24(66.7)$ & 12(33.3) & & $11(52.4)$ & 10(47.6) & \\
\hline Breast cancer at 1 st degree & & & 0.62 & & & 0.84 \\
\hline Relatives & & & & $16(47.1)$ & 18(52.9) & \\
\hline None & $376(53.0)$ & $334(47.0)$ & & $12(44.1)$ & 15(55.6) & \\
\hline Yes & $14(48.3)$ & $21(51.2)$ & & & & \\
\hline No of biopsy & & & 0.59 & & & 0.91 \\
\hline None & $370(53.0)$ & $328(47.0)$ & & $20(45.5)$ & $24(54.5)$ & \\
\hline$\geq 1$ & $20(48.8)$ & $21(51.2)$ & & $8(47.1)$ & $9(52.9)$ & \\
\hline Knowledge of risk factors & & & 0.002 & & & 0.37 \\
\hline Low & $221(58.3)$ & 158(41.7) & & $20(50.0)$ & $20(50.0)$ & \\
\hline High & 167(46.9) & 189(53.1) & & $8(38.1)$ & 13(61.9) & \\
\hline Knowledge of symptoms and signs & & & 0.004 & & & 0.17 \\
\hline Low & 155(59.8) & 104(40.2) & & $5(31.2)$ & $11(68.8)$ & \\
\hline High & $230(48.8)$ & $241(51.2)$ & & 23(51.1) & $22(48.9)$ & \\
\hline Education & & & 0.04 & & & 0.65 \\
\hline Illiterate & $47(69.1)$ & 21(30.9) & & $6(60.0)$ & $4(40.0)$ & \\
\hline Elementary & $78(53.1)$ & $69(46.9)$ & & $2(28.6)$ & $5(71.4)$ & \\
\hline High school & 182(50.3) & 180(49.7) & & $14(45.2)$ & $17(54.8)$ & \\
\hline University level & $83(51.2)$ & $79(48.8)$ & & $6(46.2)$ & $7(53.8)$ & \\
\hline Occupation & & & 0.12 & & & 0.75 \\
\hline Housewife & 292(52.7) & 292(47.3) & & 18(46.2) & 21(53.8) & \\
\hline Employee & $93(55.4)$ & $75(44.6)$ & & $4(57.1)$ & $3(42.9)$ & \\
\hline Retired & $5(29.4)$ & 12(70.6) & & $6(40.0)$ & $9(60.0)$ & \\
\hline 1st menstrual age & & & 0.09 & & & 0.47 \\
\hline$<12$ year & $48(45.3)$ & $58(54.7)$ & & $7(53.8)$ & $6(46.2)$ & \\
\hline$\geq 12$ & $335(54.0)$ & $285(46.0)$ & & $20(42.6)$ & $27(57.4)$ & \\
\hline
\end{tabular}

The $p$ values were calculated using Chi-square test

Table 6 The performance of accuracy of self-perceived risk in BC development compared with actual risk

\begin{tabular}{llllllc}
\hline Perceived risk & AUC $(\mathbf{9 5} \% \mathbf{C l})$ & $\boldsymbol{p}$ value & Sen (\%) & Sp (\%) & PPV (\%) & NPV (\%) \\
\hline 5-year risk & $0.53(0.44-0.61)$ & 0.47 & 45.9 & 54.3 & 7.6 & 92.4 \\
Lifetime risk & $0.58(0.54-0.62)$ & 0.005 & 56.0 & 55.0 & 32.7 & 76.1
\end{tabular}

$A U C$ area under the curve, Sen sensitivity, $S p$ specificity, $P P V$ positive predicted value, NPV negative predicted value

and healthcare setting; it plays a role in overestimation of breast cancer risk [31]. Selection of studies' sample from a healthcare setting such as a primary care, a hospital, or a genetic counseling clinic may be pessimism bias of breast cancer perceived risk. However, researchers also state an optimistic bias for the perceived risk had their samples from the community [31]. Health workers (physicians, midwives and nurses) that encountered with the reality of the deficiency of the pessimism opinion of the women in their daily practice can be effective to pessimism bias [31]. Future studies with comparative perceive risk of breast cancer 


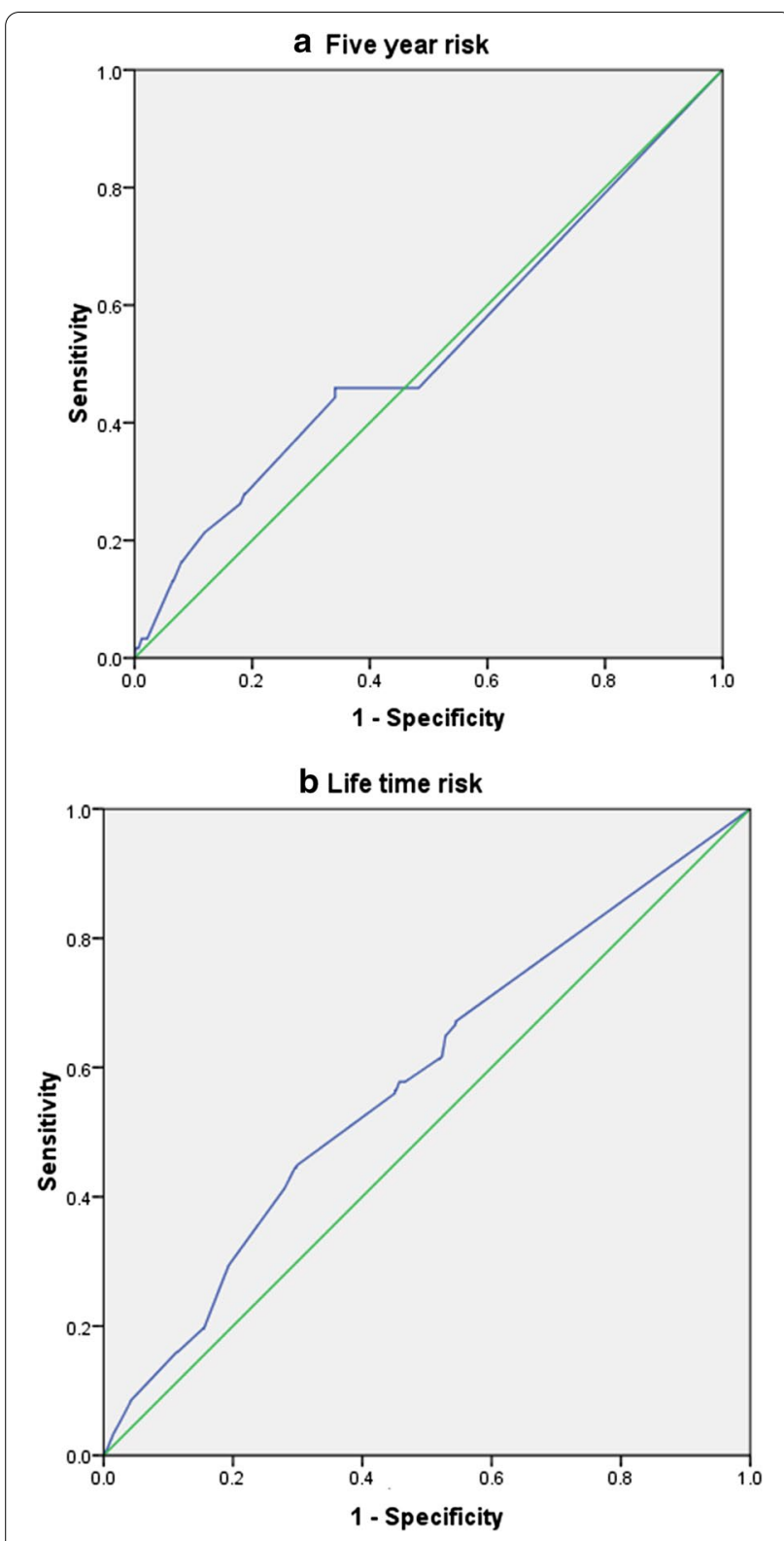

Fig. 1 ROC curves of perceived risk by visual analog scale for prediction of actual risk (a for 5 year risk and $\mathbf{b}$ for lifetime risk)

in samples from community and health centers can be provide better information.

Also pessimism of breast cancer perceived risk can be attributed to the culture of the society [20] and women worry and anxiety [32]. Researchers report that culture is an important factor in women's perceptions of breast cancer risk [20] as well as women worry and anxiety [32]. Additionally perceive measuring tools, and women's knowledge of breast cancer risk factors [20] may also be effective. Overestimated the risk of breast cancer can be a barrier to screening for health behaviors $[23,27,28]$. The probable justification for this approach bases on theory and research, it is that if the risk, (the perceived sensitivity), is high and the condition involved (breast cancer), is considered serious, the person will do less of the recommended interventions. This action is due to high fear. Overestimate the risk of breast cancer in a person, can be lead to anxiety and it is followed by the barrier of mammography [23].

Perception of the risk of disease is the main stimulus of health behaviors to prevent, diagnose, and control cancer [30]. Aligning a person's perceived and real risk of breast cancer leads to a more realistic understanding of risk [29]. As a result, it can be a motivator for appropriate health behaviors [29]. Women's optimism and pessimism about the risk of breast cancer make them evaluate their risks less and more and also it can prevent proper health behaviors [20].

The findings of the current study demonstrated that overestimated risk in younger and high education women was significantly higher than older women and lower levels of education. Jones et al. [33] in their study illustrated that younger age and higher education in women are two predictive factors in the overestimate risk of breast cancer. Also in another study, women between the ages of 30 and 39 years estimated the risk of breast cancer significantly higher than women older than 40 years [23]. More media focus on young women with breast cancer could be a possible reason for the increased perception of risk in this age group [23]. Another possible justification is the high incidence of breast cancer in younger women in recent years [27]. Breast cancer in younger women is associated with higher involvement and poor prognosis [34]. The incidence of breast cancer in Iranian women is 10 years lower than developed countries [35].These factors can justify the overestimate of the risk of breast cancer in younger women. In contrary to our observations, David et al. [34] and Cyrus-David et al. [36] reported women with higher education compared to lower education, have a more accurate estimate of the risk of breast cancer. The same is expected. But sometimes maybe a high level of awareness about a disease can give the opposite result.

\section{Limitations}

This study has limitations. Our participants did not include women $<35$ years old; therefore, we cannot generalize our findings to this younger. The real risk was calculated based on the Gail model that the baseline risk was adopted from US women. Perhaps their breast cancer risk is higher than Iranian women. Furthermore, the self-report risk perception scale is a subjective measure that may be potentially susceptible to be more pessimistic culturally. 


\section{Conclusion}

Women in the study overestimated their breast cancer risk higher than real risk. This finding illustrated a significant pessimism in breast cancer perception. Also overestimated risk in younger and high education women was significantly higher than older women and lower levels of education. Women at low or average risk of breast cancer need to have an accurate perception of their risk to avoid unnecessary anxiety and treatment. Therefore, increasing women's knowledge to perception their true risk of breast cancer should be considered by health providers.

\section{Abbreviations}

BC: Breast cancer; VAS: Visual analog scale; HBM: Health belief model; AUC : Area under the curve; Sen: Sensitivity; Sp: Specificity; PPV: Positive predicted value; NPV: Negative predicted value.

\section{Acknowledgements}

The authors acknowledge the National Institute for Medical Research Development (NIMAD), Tehran, Iran for their supports.

\section{Authors' contributions}

$\mathrm{KH}$ and $\mathrm{MN}$ have substantially contributed to the conception of study design, analysis, and drafting of the manuscript. Both authors read and approved the final manuscript.

\section{Funding}

The research reported in this publication was supported by the Elite Researcher Grant Committee under the Award Number [963287] from the National Institute for Medical Research Development (NIMAD), Tehran, Iran. The grant was received by Karimollah $\mathrm{H}$ (First author).

\section{Availability of data and materials}

To keep patients' confidentiality, the raw data would not be shared. But, it is available from the corresponding author on reasonable request, and the summary data are available in the main document.

\section{Declarations}

\section{Ethics approval and consent to participate}

The study protocol was approved by the Ethical Institutional Board of the National Institute for Medical Research Development (NIMAD), Tehran, Iran. All participants were informed about the purpose of the study and written informed consent was obtained from all participants before data collection.

\section{Consent for publication}

Not applicable.

\section{Competing interests}

The authors declare that they have no competing interests.

\section{Author details}

${ }^{1}$ Department of Biostatistics and Epidemiology, Babol University of Medical Sciences, Babol, Iran. ${ }^{2}$ Social Determinants of Health Research Center, Health Research Institute, Babol University of Medical Sciences, Babol, Iran. ${ }^{3}$ Non-Communicable Disease Research Center, Health Research Institute, Babol University of Medical Sciences, Babol, Iran.

Received: 12 July 2020 Accepted: 22 February 2021

Published online: 04 March 2021

\section{References}

1. Bidoli E, Virdone S, Hamdi-Cherif M, Tofolutti F, Taborelli M, Panato $C$, et al. Worldwide age at onset of female breast cancer: a 25-year population-based cancer registry study Ettore. Sci Rep. 2019;9:14111. https://doi.org/10.1038/s41598-019-50680-5.

2. Mulshine $J L, D^{\prime} A m i c o T A$. Issues with implementing a high-quality lung cancer screening program. CA Cancer J Clin. 2014;64(5):351-63.

3. Sharif Nia H, Behmanesh F, Kwok C, Firouzbakht M, Ebadi A, Nikpour M. Breast cancer screening beliefs questionnaire: psychometric properties of the Persian version. BMC Womens Health. 2020;20:184.

4. Mousavi SM, Gouya MM, Ramazani R, Davanlou M, Hajsadeghi N, Seddighi Z. Cancer incidence and mortality in Iran. Ann Oncol. 2009;20:556-63.

5. Taghavi IA, Fazeli Z, VahediBaghestaniPouhoseingholi MARA, Barzgar F, et al. Increased trend of breast cancer mortality in Iran. Asian Pac J Cancer Prev. 2012;13:367-70.

6. Hajian-Tilaki KO, Ahangar T. Reproductive factors associated with breast cancer risk in northern Iran. Med Oncol. 2011;28(2):441-6.

7. Hajian-Tilaki KO, Gholizadehpasha AR, Bozorgzadeh S, Hajian-Tilaki E. Body mass index and waist circumference are predictor of breast cancer risk in Iranian women. Med Oncol. 2011;28:1296-301.

8. Sweeney C, Baumgartner KB, Byers T, Giuliano AR, Herrick JS, Murtaugh MA, Slattery ML. Reproductive history in relation to breast cancer risk among Hispanic and non-Hispanic white women. Cancer Causes Control. 2008;19:391-401.

9. Shantakumar S, Terry MB, Teitelbaum SL, et al. Reproductive factors and breast cancer risk among older women. Breast Cancer Res Treat. 2007;102:365-74

10. Delavar MA, Hajian-Tilaki KO. Age at menarche in girls born from 1985 to 1989 in Mazandaran, Islamic Republic of Iran. East Mediterr Health J. 2008;14(1):90-4.

11. HajianTilaki K, Auladi S. Awareness, attitude and practice of breast cancer screening women, and the associated socio-demographic characteristics, in northern Iran. Iran J Cancer Prev. 2015;8(4):e3429.

12. Nikpour M, Hajian-Tilaki K, Bakhtiari A. Risk assessment for breast cancer development and its clinical impact on screening performance in Iranian women. Cancer Mange Res. 2019;9:10073-82.

13. Hajian-Tilaki K, Auladi S. Health belief model and practice of breast self-examination and breast cancer screening in Iranian women. Breast Cancer. 2014;21:429-34

14. Brinton LA, Brown SL, Colton T, et al. Characteristics of a population of women with breasts implants compared with women seeking other types of plastic surgery. Plast Reconstr Surg. 2000;105(3):919-27.

15. Gail MH, Brinton LA, Byar DP, Corle DK, Green SB, Schairer C, Mulvihill JJ. Projecting individualized probabilities of developing breast cancer for white females who are being examined annually. J Natl Cancer Inst. 1989;181:1879-86.

16. Chay WY, Ong WS, Tan PH, Jie Leo NQ, et al. Validation of the Gail model for predicting individual breast cancer risk in a prospective nationwide study of 28,104 Singapore women. Breast Cancer Res. 2012;14:R19.

17. Champion VL. Instrument refinement for breast cancer screening behaviors. Nurs Res. 1993;42:139-43.

18. Che Mohamed N, Moey SF, Lim BC. Validity and Reliability of Health Belief Model Questionnaire for promoting breast self-examination and screening mammogram for early cancer detection. Asian Pac J Cancer Prev. 2019;20(9):2865-73.

19. Parsa P, Kandiah M, Mohd Nasir MT, Hejar AR, Nor Afiah MZ. Reliability and validity of Champion's Health Belief Model Scale for breast cancer screening among Malaysian women. Singap Med J. 2008;49(11):897-903.

20. Chung CW, Lee SJ. Estimated risks and optimistic self-perception of breast cancer risk in Korean women. Appl Nurs Res. 2013;26(4):180-5.

21. Çeber E, Soyer MT, Ciçeklioglu M, Cimat S. Breast cancer risk assessment and risk perception on nurses and midwives in Bornova Health District in Turkey. Cancer Nurs. 2006;29(3):244-9.

22. Fehniger J, Livaudais-Toman J, Karliner L, et al. Perceived versus objective breast cancer, breast cancer risk in diverse women. J Womens Health (Larchmt). 2014;23(5):420-7.

23. Jones SC, Magee CA, Barrie LR, Iverson DC, Gregory P, Hanks EL, et al. Australian women's perceptions of breast cancer risk factors and the risk of developing breast cancer. Womens Health Issues. 2011;21(5):353-60.

24. National Cancer Institute, Breast cancer assessment tool. https://bcris ktool.cancer.gov/calculator.html. 
25. Heidari Z, Feizi A. Transcultural adaptation and validation of the Persian version of the breast cancer awareness measure (BCAM) questionnaire. Cancer Med. 2018;7(10):5237-51.

26. Linsell L, Forbes LJL, Burgess C, Kapari M, Thurnham A, Ramirez AJ. Validation of a measurement tool to assess awareness of breast cancer. Eur J Cancer. 2010;46(8):1374-81.

27. Aduayi VA, Onayade AA, Aduayi OS, Odu OO. Relating self-perceived with objective risk for breast cancer among women in rural and urban communities in southwestern Nigeria. Int J Med Sci Public Health. 2015;4(10):1.

28. Calvocoressi L, Kasl SV, Lee CH, Stolar M, Claus EB, Jones BA. A prospective study of perceived susceptibility to breast cancer and nonadherence to mammography screening guidelines in African American and White women ages 40 to 79 years. Cancer Epidemiol Prevent Biomark. 2004;13(12):2096-105.

29. Katapodi MC, Dodd MJ, Facione NC, Humphreys JC, Lee KA. Why some women have an optimistic or a pessimistic bias about their breast cancer risk: experiences, heuristics, and knowledge of risk factors. Cancer Nurs. 2010;33(1):64-73.

30. Banegas MP, Püschel K, Martínez-Gutiérrez J, Anderson JC, Thompson B. Perceived and objective breast cancer risk assessment in Chilean women living in an underserved area. Cancer Epidemiol Prevent Biomark. 2012;21(10):1716-21.
31. KartalOzcakarHatipoglu MNS, Neslisah TanDilek Gulda MA. Breast cancer risk perceptions of Turkish women attending primary care: a cross-sectional study. BMC Women's Health. 2014;14:152.

32. Lee JM, Lowry KP, Chubiz JEC, Swan JS, Motazedi T, Halpern EF, et al. Breast cancer risk, worry, and anxiety: effect on patient perceptions of false-positive screening results. The Breast. 2020;50:104-12.

33. Jones SC. Coverage of breast cancer in the Australian print media-does advertising and editorial coverage reflect correct social marketing messages? J Health Commun. 2004;9(4):309-25.

34. Davids SL, Schapira MM, McAuliffe TL, Nattinger AB. Predictors of pessimistic breast cancer risk perceptions in a primary care population. J Gen Intern Med. 2004;19(4):310-5.

35. Yazdani-Charati R, Hajian-Tilaki K, Sharbatdaran M. Comparison of pathologic characteristics of breast cancer in younger and older women. Caspian J Intern Med. 2019;10910:42-7.

36. Cyrus-David MS. Knowledge and accuracy of perceived personal risk in underserved women who are at increased risk of breast cancer. J Cancer Educ. 2010;25(4):617-23.

\section{Publisher's Note}

Springer Nature remains neutral with regard to jurisdictional claims in published maps and institutional affiliations.
Ready to submit your research? Choose BMC and benefit from:

- fast, convenient online submission

- thorough peer review by experienced researchers in your field

- rapid publication on acceptance

- support for research data, including large and complex data types

- gold Open Access which fosters wider collaboration and increased citations

- maximum visibility for your research: over $100 \mathrm{M}$ website views per year

At BMC, research is always in progress.

Learn more biomedcentral.com/submissions 